\title{
ESTUDO DE SIMULAÇÃO DA RECONFIGURAÇÃO DO LAYOUT LOGÍSTICO PARA REDUÇÃO DE CUSTOS E ELIMINAÇÃO DE GARGALOS
}

\section{STUDY SIMULATION TO RECONFIGURE THE LOGISTICS LAYOUT FOR COST REDUCTION AND BOTTLENECKS ELIMINATION.}

\author{
Daniel Bovo \\ Universidade de São Paulo - USP-São Paulo - Brasil \\ danielbovo@hotmail.com
}

\begin{abstract}
Resumo
Este artigo apresenta um estudo de caso de mudança de layout nas operações logísticas de uma empresa do setor industrial e da simulação de cenários, com seus impactos e propostas de soluções. Devido ao compartilhamento de recursos no processo de carregamento $e$ descarregamento em uma das plantas da empresa, o tempo que cada veículo permanece dentro da unidade (TPV) estava excessivamente alto, impactando no prazo de atendimento ao cliente $e$ gerando volumosos gastos com o pagamento de diárias às transportadoras em função deste tempo elevado, cenário este que poderia piorar consideravelmente devido à expectativa de crescimento da produção para os próximos anos. Assim, a finalidade deste estudo é definir através da simulação qual a melhor configuração dos fluxos inbound e outbound para suportar o crescimento projetado de vendas/operações nos próximos cinco anos e reduzir o tempo de permanência dos veículos dentro planta. Através da simulação, foi possível desenvolver alguns cenários com mudanças de layouts e também com a adição de novos recursos, que geraram reduções de até $70 \%$ no TPV e consequentemente nos gastos com diárias, tendo este trabalho como resultado uma série de recomendações para a melhoria da operação.
\end{abstract}

Palavras-chave: planejamento de capacidade; modelagem; simulação logística; cadeia de suprimentos.

\section{Introdução}

Empresa do setor metalúrgico, aqui denominada de "Alpha", é uma das maiores da América Latina, com plantas no Brasil e exterior. Fornece produtos de alta qualidade para os mais variados setores da indústria e da construção civil.

Nos últimos anos, o setor metalúrgico vem sofrendo fortes reduções na margem de EBITDA (Earnings Before Interest, Taxes, Depreciation and Amortization) em função da grande concorrência interna e do aumento de produtos importados, exercendo uma grande pressão sobre as organizações para operarem com excelência e ao menor custo possível. Adicional a isso, este mesmo mercado, exige cada vez mais de seus fornecedores em relação à disponibilidade de 
produtos e prazos de entregas menores. É nesse cenário que a revisão da estratégia logística se faz mais importante para que a empresa possa se manter competitiva nesse setor.

No Brasil, a operação da empresa é composta por três usinas de produção (produto acabado), dois centros de distribuição, dez filiais e seis unidades de produção upstream, espalhadas por todas as regiões do país.

Cada uma das três unidades de produção, no geral, é especializada em determinadas famílias de produtos, sendo que a maior delas (objeto de estudo deste trabalho), além de produzir produtos acabados que são enviados diretamente para clientes e para abastecimento dos centros de distribuição e filiais, ainda fornece material em processo para as demais plantas.

Todo material que chega ou sai desta unidade é transportado via modal rodoviário, e que em função do elevado volume movimentado, gera um fluxo muito grande de caminhões dentro da usina, ocasionando muitas filas, tanto para o processo de carga (outbound) e descarga (inbound).

Com o aumento de produção e venda previstos para os próximos anos, esta planta, que hoje já está bastante engargalada quanto à utilização de recursos, terá ainda mais problemas logísticos internos para recebimento, movimentação e expedição de materiais devido à falta de capacidade dos mesmos e a concentração de faturamento em determinados períodos.

A planta opera no regime 7 × 24 horas e não para suas operações em nenhum dia do ano. Hoje, o tempo total de carga e descarga de veículos, aqui denominado de TPV (tempo de permanência de veículo), além de possuir uma média em horas bastante elevada, o desvio padrão dessa operação é igualmente alto, o que mostra a grande instabilidade deste processo, conforme Figura 1.

Figura 1 - Tempo de Permanência de Veículos
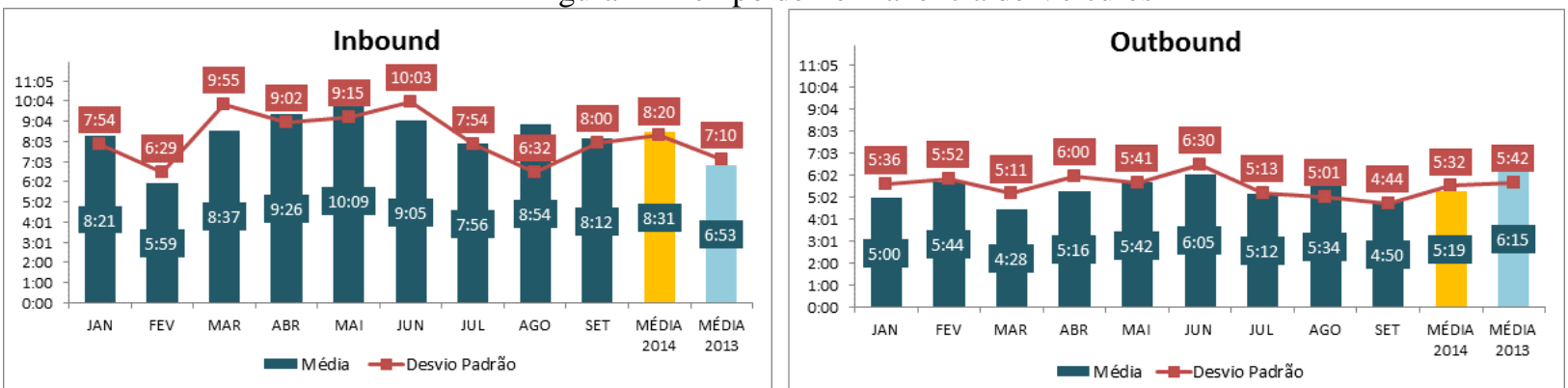

Fonte: Pesquisa de campo (2014)

Em decorrência deste TPV elevado, é gerado um gasto de alguns milhões de reais com o pagamento de diária para as transportadoras, que ocorrem quando um veículo permanece acima de determinada quantidade de horas (acordadas em contrato) dentro da planta. Um agravante a esta situação é a possibilidade da entrada em vigor de uma nova lei, chamada de Lei do Motorista, que pode fazer com que estes gastos com diárias tripliquem. 
Atualmente, a planta possui uma configuração onde os fluxos inbound e outbound se misturam e compartilham os mesmos recursos durante todo o processo em que os veículos estão dentro da unidade (cadastro, estacionamento, preparação do veículo e pesagem/balança, tanto na entrada como na saída), o que torna a operação ainda mais complicada.

Assim, dado este cenário, a operação da planta irá se tornar mais complexa e demorada, fazendo com que o tempo que cada veículo permanece dentro da planta aumente consideravelmente, tanto para descarregar matéria prima quanto carregar produto acabado, ocasionando além de gastos com diárias, perda no nível de serviço no atendimento ao cliente, perda de competitividade no mercado e perda de atratividade da empresa como embarcador (aumentando a pressão para aumento do custo do frete).

O objetivo deste trabalho é definir através da simulação qual a melhor configuração para os fluxos inbound e outbound para suportar o crescimento projetado de vendas/operações nos próximos 5 anos e reduzir o tempo de permanência dos veículos dentro planta.

Como parte deste plano, serão estudados e identificados os recursos críticos de todo o processo logístico de dentro da planta (pontes rolantes, balança, docas, equipamentos de descarga, etc) que são ou serão gargalos nos próximos anos, e propor alternativas para além de resolvê-los permitir a total utilização da capacidade de expedição, reduzir o TPV e consequentemente os custos com diárias pagas aos transportadores.

Inicialmente buscou-se ajuda de consultorias especializadas no assunto, porém, dado o elevado custo para a realização deste estudo, a empresa decidiu investir na capacitação interna para o desenvolvimento do projeto. Estruturou-se uma equipe com pessoas da área de logística, suprimentos, industrial e comercial.

Este artigo está organizado em seis seções. Esta primeira parte é a Introdução, onde são apresentados a empresa, a operação e o problema em questão. A segunda seção tratará da fundamentação teórica do trabalho. A seção três descreve a metodologia utilizada para o estudo e solução do problema. Na quarta, será feito o aprofundamento do tema e serão apresentados os dados da operação. A seção cinco é dedicada à discussão dos resultados obtidos com a modelagem e simulação da operação. Por fim, serão apresentadas as conclusões e próximos passos do projeto.

\section{Fundamentação teórica}

Nesta parte serão discutidos três pontos que influenciam diretamente na solução do problema mencionado na primeira seção. São eles:

- Planejamento de capacidade;

- Simulação de operações logísticas; 
- Teoria das restrições.

Uma vez discutidas as questões referentes ao planejamento de capacidade, será apresentada uma revisão sobre simulação de sistemas, que é a base do desenvolvimento deste trabalho, e por fim, será discutido a respeito da teoria das restrições, que trata da identificação e eliminação de gargalos nas operações.

\subsection{Planejamento de capacidade}

Por definição, capacidade de uma operação é o máximo nível de atividade de valor adicionado em determinado período de tempo que o processo pode realizar sob condições normais de operação (SLACK et al., 2009).

O planejamento de capacidade é de fundamental importância para o futuro das organizações de modo que um planejamento incorreto pode acarretar em dois extremos indesejáveis. Por um lado, a empresa pode não ter capacidade suficiente para atender a demanda, levando a perdas de faturamento e de nível de serviço, e por outro, pode-se ter capacidade ociosa, podendo gerar o aumento dos custos operacionais.

O planejamento de capacidade costuma ser realizado em dois níveis, correspondendo a decisões estratégicas ou táticas. O primeiro deles é o estratégico e se caracteriza por ser de longo prazo. Neste nível a empresa decide quais serão os investimentos a serem feitos em maquinário, instalações, infraestrutura, entre outros, de modo a antecipar a necessidade de capacidade de recursos que precisam de um prazo relativamente longo (meses ou anos) para sua implantação/obtenção (CORRÊA et al., 2010). Neste modelo, além do tempo elevado de mudança, em geral, é exigido grande dispêndio de capital para sua realização.

O segundo nível de decisão é de caráter tático/operacional, com enfoque em necessidades de curto prazo, que incluem, por exemplo, o planejamento da força de trabalho, os volumes de estoques e o uso diário de maquinário. Neste nível, pretende-se antecipar necessidades de capacidade de recursos que exijam poucos meses ou semanas para sua mobilização.

Dentro do supply chain, as restrições de capacidade podem estar localizadas em qualquer elo da cadeia, desde o fornecimento de matéria prima, produção, até a distribuição, e que independente de onde esteja, restringe a capacidade global do sistema. Desse modo, é preciso rastrear onde estão estes gargalos, podendo ser aplicada a teoria das restrições para esta análise. E pensando em um horizonte maior de tempo, estimar quais serão as necessidades de capacidade de longo prazo.

A teoria das restrições (assunto que será abordado na seção 2.3) analisa o como administrar da melhor forma a capacidade já existente e as operações cotidianas, ao passo que as decisões de 
longo prazo devem ser tomadas para planejar a capacidade de cada processo (KRAJEWSKI et al., 2009).

\subsection{Simulação de operações logísticas}

Simulação é um processo de experimentação com a criação de modelos detalhados de um sistema real com o intuito de determinar como este mesmo sistema responderá a mudanças em sua estrutura, ambiente ou condições de contorno (BATEMAN et al, 2013). Modelos de simulação são representações físicas, matemáticas ou lógicas de um sistema, processo ou fenômeno (SAHINOGLU, 2013).

A técnica de simulação permite testes do tipo what if, no qual se alteram um ou mais parâmetros do modelo e observam-se os resultados de saída. Em cenários de modelagem, o modelo é submetido a entradas conhecidas, obtidas através da análise de dados históricos ou por meio do estudo de tempos e movimentos, e que após a simulação computacional, retorna as respostas de saída do modelo (SELLITTO, 2009).

Segundo Ballou (2006), a técnica de simulação computacional é adequada para avaliar a dinâmica dos fluxos de informação e materiais dentro de uma operação logística. Através da simulação é possível testar inúmeros cenários de modo rápido e de baixo custo quando comparado a experimentos reais. Desse modo, é possível testar alternativas de abastecimento ou distribuição de produtos e verificar os possíveis resultados nos custos de produção e no nível de serviço.

De acordo com Oliveira (2004), o ambiente de simulação de uma cadeia de suprimentos (CS) considera a dinâmica da simulação e deve respeitar dois aspectos importantes, que são a lógica de coordenação da simulação e a representação das regras de negócio da empresa e da CS. Nestes tipos de simulações, é possível analisar a logística de armazéns, fábricas e sistemas de distribuição, obtendo respostas de alternativas para layouts, fluxo de materiais, meios de transportes, alocação de recursos e pessoas, entre outros.

Segundo Longo, a grande utilização da simulação na indústria e na logística é para as empresas se manterem competitivas, testarem novas estratégias de produção e gerenciamento e para se obter um melhor entendimento sobre o comportamento de sistemas complexos.

Segundo Harrel et al.(2004), não existem regras sobre como se deve conduzir um projeto de simulação, porém existem alguns passos que são recomendados e que podem ajudar na condução adequada do mesmo:

- Definição do problema e estabelecimento dos objetivos: definição do propósito do projeto de simulação e detalhamento do escopo. Planejamento da necessidade de recursos, cronograma e orçamento; 
- Coleta e análise de dados: obtenção de todas as informações necessárias para a construção do modelo e entendimento do problema. Identificar, reunir e analisar os dados do sistema a ser modelado;

- Desenvolvimento do modelo: construção do modelo de simulação do sistema a ser analisado. Inicia-se com um desenho conceitual do sistema, com crescentes níveis de detalhes ao passo que a modelagem avança;

- Verificação: verificar se o modelo se comporta de acordo com o que o modelador pretendia. A verificação pode ser realizada rodando-se a simulação e monitorando-se de perto sua operação, como por exemplo, utilizando uma velocidade de animação suficientemente baixa de modo a analisar o movimento de cada entidade do sistema;

- Validação: assegurar que o modelo reflita com credibilidade o comportamento real do sistema e que possa trazer solução aos problemas identificados;

- Experimentação: definir a extensão necessária de tempo e executar a simulação para cada um dos cenários propostos e analisar os resultados;

- Análise dos resultados e apresentação: documentação dos cenários e apresentação dos resultados obtidos, além da listagem das alternativas encontradas e das recomendações a respeito do que se deve ser feito.

Cada um dos passos acima citados não precisa ter sido terminado para que se possa passar a etapa seguinte. O processo de simulação é dinâmico, e a decisão de um detalhamento maior deve ser regida pelos objetivos e restrições do problema, bem como, por uma análise de sensibilidade, para determinar se um refinamento adicional trará resultados mais significativos.

Como mencionado, a simulação busca refletir com o maior realismo possível o sistema em estudo. Porém, como mencionado por Iannoni e Morabito (2002), na prática sabe-se que existem alguns aspectos externos que podem influenciar nos resultados da simulação (como por exemplo, quebra de equipamentos, problemas industriais, fenômenos climáticos, entre outros) que são bastante difíceis de serem refletidos nos modelos, e que associado a algumas simplificações adotadas, fazem com que o resultado do modelo varie um pouco da realidade.

\subsection{Teoria das restrições}

A teoria das restrições (ToC - Theory of Constraints) teve início na década de 80 com o físico israelense Eliyahu M. Goldratt, o qual desenvolveu uma filosofia baseada em princípios em que todo sistema, não importando quão complexo ele possa ser, é limitado em sua capacidade de gerar maiores resultados devido a um pequeno número de variáveis, denominadas de restrições, e que ao eliminá-las, pode-se obter grandes incrementos nos volumes de produção. 
Assim, restrição ou gargalo, pode ser qualquer fator que impeça um sistema de atingir um nível melhor de desempenho. Elas podem ter diversas origens, podendo ser restrições físicas, como por exemplo, um equipamento ou a falta de material, ou podem ser também restrições de ordem gerencial, como procedimentos, políticas e normas (COX; SPENSER, 2002).

Para a identificação das restrições de um sistema, Goldratt desenvolveu o chamado processo de focalização de cinco etapas (GOLDRATT; COX, 2003; MOELLMANN, 2008) que consistem em:

1) Identificar a restrição do sistema: identificar as restrições que podem impedir que a organização obtenha mais de sua meta em uma unidade de tempo;

2) Explorar a restrição do sistema: decidir como explorar as restrições do sistema para tirar o máximo proveito das mesmas;

3) Subordinar tudo o mais à decisão acima: subordinar todas as demais atividades à restrição do sistema para suportar a decisão tomada acima;

4) Elevar a restrição do sistema: aumentar a capacidade para um nível mais alto;

5) Se na etapa anterior a restrição for quebrada, volte à etapa 1: caso a restrição tenha sido eliminada na etapa 4, volte para a etapa 1 para evitar que a inércia interrompa o processo de aprimoramento contínuo.

Outro conceito introduzido por Goldratt é o do tambor-pulmão-corda (do inglês drumbuffer-rope), que é uma técnica utilizada para gerenciar e programar os recursos a fim de maximizar o ganho das empresas (COX; SPENSER, 2002):

- Tambor: marca o ritmo de produção determinado pela restrição. Isso significa que todos os demais recursos produtivos são sincronizados com a programação da restrição.

- Pulmão: é responsável por proteger a restrição contra interrupções, evitando perdas em todo o sistema. Consiste de um "estoque" de tempo ou material para sustentar o ganho e/ou desempenho dos prazos de entrega.

- Corda: processo de comunicação entre o gargalo e o processo final que controla ou limita o material liberado no sistema para alimentar a restrição de acordo com a sua programação.

Assim, através da utilização dos conceitos da ToC ensinados por Goldratt e da técnica de simulação, foi possível identificar e atuar nos gargalos da operação da planta da empresa em análise e assim propor soluções para, além de melhorar o fluxo de veículos, reduzir filas de espera e aumentar a produtividade dos recursos, conforme será apresentado no estudo de caso deste artigo.

\section{Método}


A metodologia a ser utilizada neste estudo será a de simulação computacional através de um software especializado, no caso o ProModel, com análise de cenários do tipo What if, para que se possa obter a melhor resposta do sistema. Para pequenas análises de melhoria de alguns subsistemas do processo, foi utilizado o software What's Best, que roda direto no Excel, facilitando as análises.

Para a obtenção de dados, foram utilizadas basicamente três fontes: o banco de dados do ERP da empresa (no caso, o SAP), dados coletados em estudo de tempos e movimentos, e entrevistas realizadas com funcionários das diversas áreas envolvidas (operacional, industrial, logística, comercial).

O projeto foi estruturado conforme demonstrado na Figura 2.

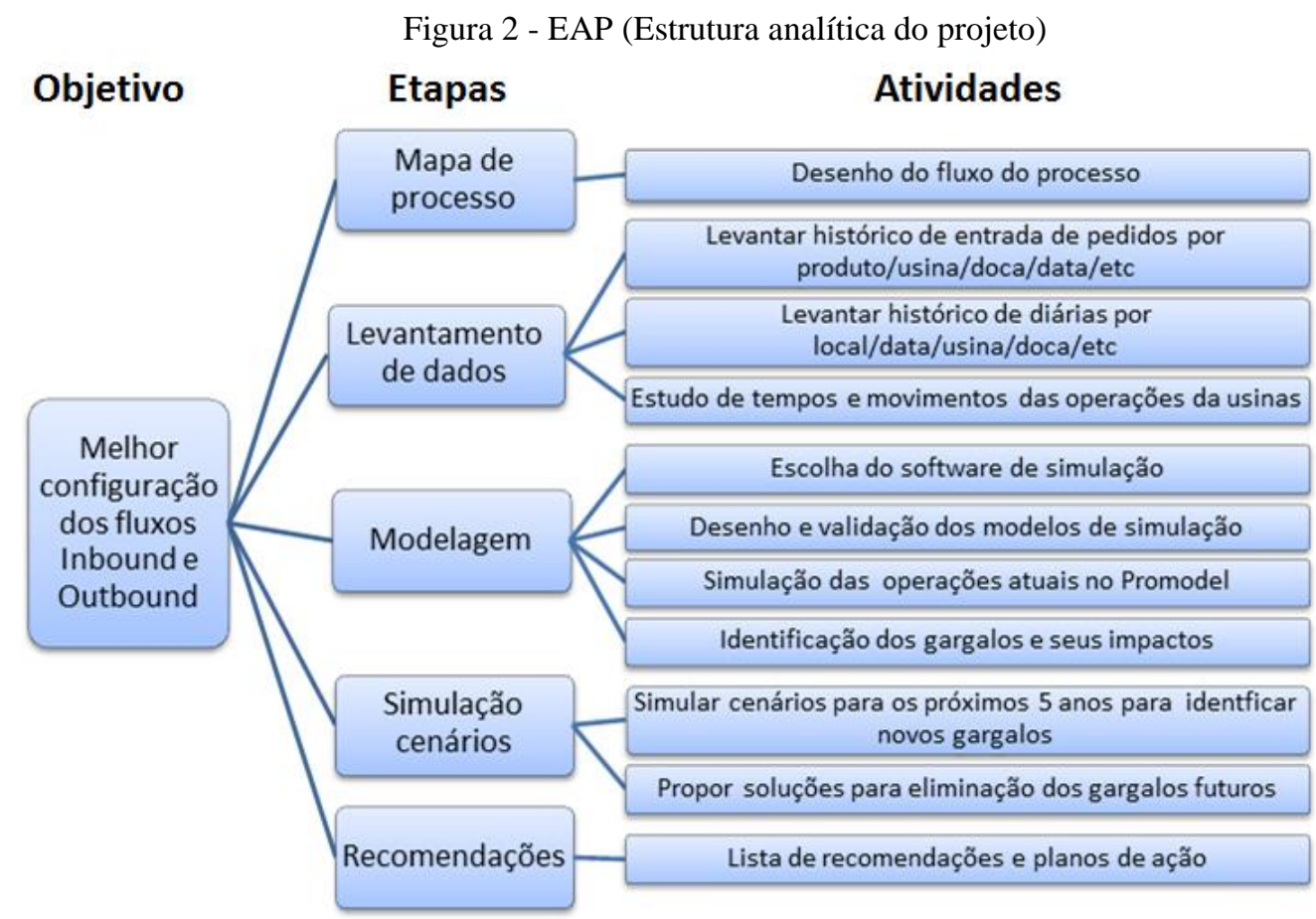

Fonte: Autoria própria (2014)

\section{Projeto de Simulação}

\subsection{Mapeamento do Processo}

Dado que o objetivo deste trabalho é definir qual a melhor configuração para os fluxos inbound e outbound para suportar o crescimento projetado de vendas/operações nos próximos 5 anos, bem como reduzir o TPV, o ponto de partida foi o desenho do fluxo de carga e descarga que todo caminhão deveria percorrer dentro da unidade, e a partir dele, definir o plano de medição dos tempos e movimentos de cada uma das operações envolvidas.

Na Figura 3, é apresentado um layout simplificado do fluxo logístico da unidade. Conforme já mencionado, tais fluxos, inbound e outbound, atualmente compartilham os mesmos recursos dentro da planta, exceto pelo processo de carregamento ou descarregamento. 
Figura 3 - Fluxo logístico do processo de carga e descarga

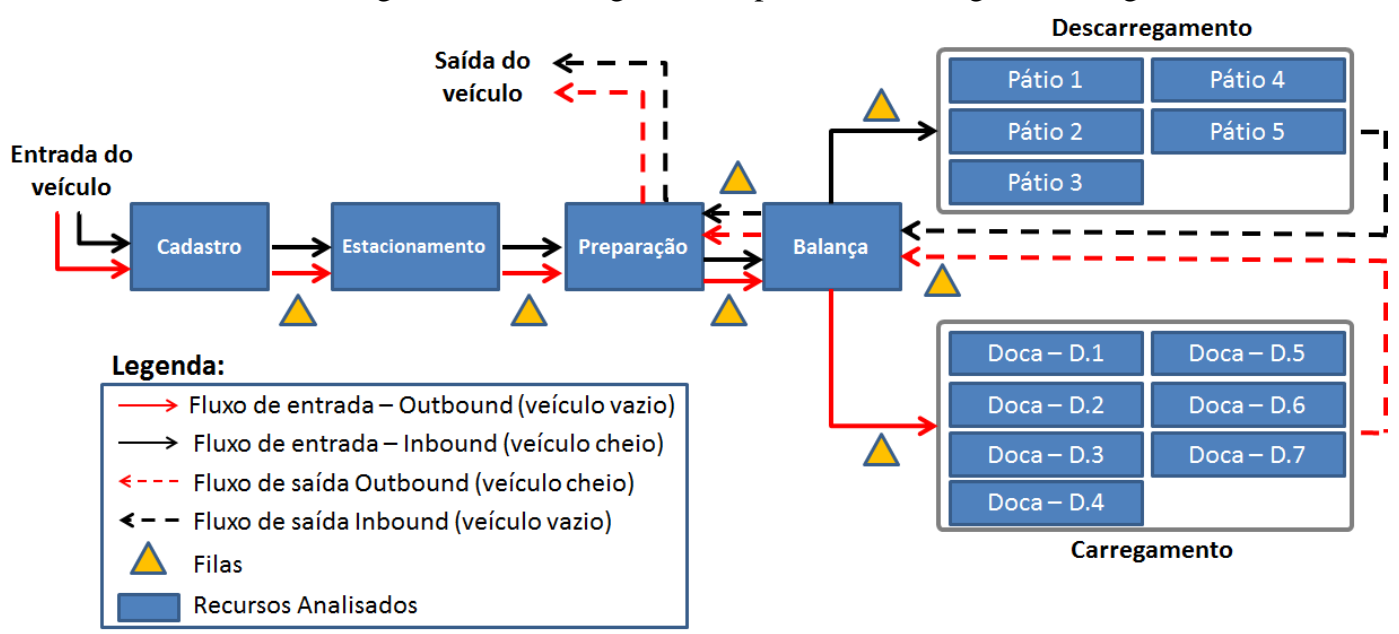

Fonte: Autoria própria (2014)

Abaixo é descrito cada um dos processos apresentados na Figura 3, com detalhes das atividades, equipamentos e quantidades de locais (no caso das docas de carga e descarga).

- Cadastro: responsável pelo cadastramento do veículo e de disponibilizar o mesmo para o sistema.

- Preparação: todo veículo que entra ou sai da planta passa por uma preparação específica, como a de desenlonamento e/ou colocação de calços para os veículos que estão entrando na planta, e de amarração e enlonamento para os veículos que deixarão a planta com produto acabado.

- Balança: pesagem do veículo, tanto na entrada como na saída.

- Docas de carregamento: sete docas de carregamento, cada uma delas dedicada a um tipo de família de produto e com equipamentos específicos para tal, como pontes rolantes com eletroímã, pontes rolantes com ganchos ou empilhadeiras.

- Docas de descarregamento: cinco pátios de descarga, onde podem ser utilizadas pontes rolantes, escavadeiras com garra ou escavadeiras com eletroímã.

Uma vez que o veículo foi cadastrado no sistema, ele fica aguardando no estacionamento até que o mesmo seja chamado para entrar na planta, sendo que para tal, ele necessariamente tem que passar pela preparação e pela balança. Seguindo o processo, o veículo segue para as docas de carga ou descarga, e depois novamente a balança e a preparação para sua saída da unidade.

\subsection{Coleta de dados}

Para medir a capacidade de cada um dos recursos apresentados no fluxo da Figura 3, estruturou-se um plano para a tomada dos tempos de toda a operação envolvida em cada um deles. O plano foi elaborado em função do tipo de processo a ser realizado (inbound ou outbound), do tipo de produto, do tipo de caminhão e do tipo de equipamento utilizado. 
Como já mencionado, parte das informações foram obtidas diretamente do sistema da empresa e dos bancos de dados existentes, entretanto, para parte delas, foi preciso ir a campo e coletar estes novos dados.

Para garantir a coleta de uma amostragem confiável e que refletisse a realidade da operação e de suas incertezas e interferências, as medições (detalhadas na Figura 4) foram realizadas aleatoriamente em dias e horários diferentes, conforme demonstrado na Figura 5, levando assim, algumas semanas para a finalização das mesmas.

Na Figura 4, observa-se o como foram divididas as medições e também o número de amostras em cada etapa do processo, e na Figura 5 é demonstrado um exemplo da planilha de dados resultante das medições do tempo de carregamento na doca do acabamento 1 .

Figura 4 - Número de medições

\begin{tabular}{c|c|c|c|c|c|} 
& \multicolumn{3}{c}{ Cadastro } & \multicolumn{2}{c}{ Pátios de descarga } \\
Plano de & 26 & 26 & 57 & 97 & 55 \\
\cline { 2 - 6 } Medições & \multicolumn{3}{c}{ Preparação } & \multicolumn{5}{c}{ Docas de carregamento }
\end{tabular}

Fonte: Autoria própria (2014)

Figura 5 - Estrutura da tabela de dados das medições

\begin{tabular}{|c|c|c|c|c|c|c|c|c|c|c|c|c|}
\hline \multicolumn{6}{|c|}{ Informações do processo } & \multicolumn{3}{|c|}{ Características da operação } & \multicolumn{4}{|c|}{ Tempos da operação } \\
\hline Unidade & Processo2 & Doca & Responsável & $\begin{array}{c}\text { Data da } \\
\text { medição }\end{array}$ & $\begin{array}{l}\text { Hora da } \\
\text { medição }\end{array}$ & Tipo do Veículo & $\begin{array}{l}\text { Número de } \\
\text { operadores }\end{array}$ & $\begin{array}{c}\text { Equipamentos } \\
\text { utilizados }\end{array}$ & Tempo Total & Interferência & $\begin{array}{l}\text { Tirada de } \\
\text { produção }\end{array}$ & $\begin{array}{c}\text { Tempo } \\
\text { Operação }\end{array}$ \\
\hline Planta 1 & Carregamento & Acabamento 1 & Diogo & $14 / 06 / 2014$ & $08: 45: 00$ & Carreta 5E-27 & 1 & Pte. Eltro Imã & $00: 51: 50$ & $00: 11: 20$ & $00: 11: 20$ & $00: 40: 30$ \\
\hline Planta 1 & Carregamento & Acabamento 1 & Michel & $15 / 06 / 2014$ & $16: 40: 00$ & Vanderléia 5E-36 & 2 & Pte. Eltro Imã & $00: 35: 22$ & & & $00: 35: 22$ \\
\hline Planta 1 & Carregamento & Acabamento 1 & Diogo & $10 / 07 / 2014$ & $15: 10: 00$ & Carreta 5E-27 & 2 & Pte. Eltro Imã & 01:22:43 & $00: 52: 39$ & $00: 47: 38$ & $00: 30: 04$ \\
\hline Planta 1 & Carregamento & Acabamento 1 & Michel & $14 / 06 / 2014$ & 15:20:00 & Carreta $6 \mathrm{E}-30$ & 1 & Pte. Rolante & $00: 49: 19$ & & & $00: 49: 19$ \\
\hline Planta 1 & Carregamento & Acabamento 1 & Diogo & $16 / 06 / 2014$ & $16: 40: 00$ & Carreta 5E-27 & 2 & 2 Pte. Rolantes & $00: 55: 47$ & $00: 21: 22$ & $00: 16: 22$ & $00: 34: 25$ \\
\hline Planta 1 & Carregamento & Acabamento 1 & Diogo & $22 / 06 / 2014$ & $10: 00: 00$ & Carreta $6 \mathrm{E}-30$ & 2 & Pte. Rolante & $00: 52: 25$ & $00: 19: 58$ & & $00: 32: 27$ \\
\hline Planta 1 & Carregamento & Acabamento 1 & Diogo & $11 / 07 / 2014$ & 19:55:00 & Carreta 5E-27 & 3 & Pte. Rolante & $00: 22: 58$ & & & $00: 22: 58$ \\
\hline Planta 1 & Carregamento & Acabamento 1 & Diogo & $05 / 07 / 2014$ & $22: 15: 00$ & Carreta 5E-27 & 3 & Pte. Rolante & $00: 53: 18$ & $00: 24: 02$ & & $00: 29: 16$ \\
\hline
\end{tabular}

Fonte: Pesquisa de campo (2014)

Como as medições foram feitas por várias pessoas, foi elaborada uma ficha padronizada com todas as atividades que deveriam ser medidas, além de campos de observação para descrever interferências ocorridas durante este processo, conforme apresentado na Figura 6. Estas interferências afetam a capacidade de carregamento ou descarregamento, impactando na capacidade total do sistema. 
Figura 6 - Ficha padronizada para a tomada de tempos de cada operação

\begin{tabular}{|c|c|c|c|c|c|c|c|c|c|c|c|c|}
\hline \multirow{4}{*}{ Outbound } & & Medição 1 & Medição 2 & Medição 3 & Medição 4 & Medição 5 & Medição 6 & Medição 7 & Medição 8 & Medição 9 & Medição 10 & \\
\hline & Responsável & Diogo & Diogo & Diogo & Cláudio & Paulo & Paulo & Paulo & Paulo & Diogo & Diogo & \\
\hline & Data & $14 / 06 / 2014$ & $14 / 06 / 2014$ & $28 / 06 / 2014$ & $15 / 06 / 2014$ & $15 / 06 / 2014$ & $15 / 06 / 2014$ & $05 / 07 / 2014$ & $05 / 07 / 2014$ & $10 / 07 / 2014$ & $13 / 022014$ & \\
\hline & Hora & $08: 45$ & $15: 15$ & $10: 25$ & $04: 27$ & 11:00 & $16: 40$ & $17: 00$ & $22: 44$ & $15: 10$ & $21: 40$ & \\
\hline \multirow{5}{*}{ Planta 1} & Doca & Doca D.1 & Doca D.1 & Doca D.1 & Doca D.1 & Doca D.1 & Doca D.1 & Doca D.1 & Doca D.1 & Doca D.1 & Doca D.1 & \\
\hline & Placa Veículo & $X X X-1234$ & XXX-1234 & XXX-1234 & XXX-1234 & XXX-1234 & $X X X-1234$ & $X X X-1234$ & $X X X-1234$ & $X X X-1234$ & $X X X-1234$ & \\
\hline & Tipo do Veículo & Carreta 5E-27 & Carreta 5E-27 & Carreta 6E-30 & Carreta 5E-27 & Carreta 5E-27 & Car. Vand. 5E-3t & Carreta 6E-30 & Car. Vand. 5E-36 & Carreta 5E-27 & Carreta 5E-27 & \\
\hline & $\mathrm{N}^{\circ}$ Pessoas & 1 & 2 & 1 & 1 & 2 & 2 & 2 & 2 & 2 & 2 & \\
\hline & Equipamentos & Pte. Rolante & Pte. Eltro Imã & Pte. Eltro Imã & Pte. Rolante & Pte. Rolante & Pte. Eltro Imã & Pte. Rolante & Pte. Rolante & Pte. Eltro Imã & Pte. Rolante & \\
\hline \multicolumn{2}{|c|}{ Medições } & Carro 1 & Carro 2 & Carro 3 & Carro 4 & Carro 5 & Carro 6 & Carro 7 & Carro 8 & Carro 9 & Carro 10 & Média \\
\hline \multicolumn{2}{|c|}{ Manobra do veículo } & 00:02:43 & 00:01:56 & 00:01:36 & 00:00:47 & 00:02:40 & 00:04:11 & $00: 02: 58$ & 00:01:39 & 00:03:28 & 00:02:22 & 00:02:26 \\
\hline \multicolumn{2}{|c|}{$\begin{array}{l}\text { Verificação da remessa } \\
\text { /Localização do material }\end{array}$} & $00: 04: 32$ & $00: 26: 37$ & - & $00: 02: 22$ & $00: 03: 30$ & $00: 08: 52$ & 00:01:02 & $00: 02: 24$ & $00: 25: 40$ & $00: 28: 41$ & 00:11:31 \\
\hline \multirow{2}{*}{\multicolumn{2}{|c|}{ 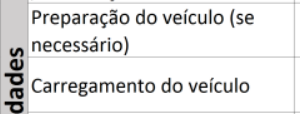 }} & 00:00:00 & 00:01:35 & 00:00:00 & 00:03:04 & 00:00:00 & 00:00:00 & 00:00:00 & 00:00:00 & 00:00:00 & 00:00:00 & 00:00:28 \\
\hline & & $00: 42: 39$ & 00:31:05 & 01:28:15 & $00: 53: 15$ & 01:01:51 & $00: 20: 30$ & $01: 31: 37$ & 01:26:08 & 00:50:06 & $00: 25: 12$ & 00:55:04 \\
\hline \multicolumn{2}{|c|}{$\begin{array}{l}\sum_{\text {Amarração do veículo (se }} \text { Amecessário) } \\
\end{array}$} & 00:00:00 & 00:00:00 & 00:00:00 & 00:00:00 & 00:00:00 & 00:00:00 & $00: 00: 38$ & 00:00:00 & 00:01:08 & 00:00:00 & 00:00:11 \\
\hline \multicolumn{2}{|c|}{$\begin{array}{l}\text { Finalização do carregamento } \\
\text { (documentação) }\end{array}$} & 00:00:39 & 00:01:31 & 00:01:02 & $00: 02: 14$ & $00: 00: 57$ & 00:00:39 & $00: 00: 32$ & 00:10:09 & 00:00:51 & 00:02:07 & 00:02:04 \\
\hline \multicolumn{2}{|c|}{ Liberação do veículo } & $00: 01: 17$ & 00:01:06 & $00: 00: 31$ & $00: 01: 23$ & 00:02:37 & 00:01:10 & $00: 01: 22$ & 00:01:01 & 00:01:30 & $00: 00: 26$ & 00:01:14 \\
\hline \multicolumn{2}{|c|}{ Total } & 00:51:50 & 01:03:50 & 01:31:24 & 01:03:05 & 01:11:35 & $00: 35: 22$ & 01:38:09 & 01:41:21 & $01: 22: 43$ & $00: 58: 48$ & 01:11:49 \\
\hline \multicolumn{2}{|c|}{ Observação / Interferencia } & - & - & Tirar produção & - & - & - & Tirar produção & Tirar produção & - & - & \\
\hline Temp & la obs. & - & - & $00: 16: 32$ & - & - & - & $00: 22: 23$ & $00: 18: 54$ & - & - & \\
\hline
\end{tabular}

Fonte: Pesquisa de campo (2014)

A capacidade de cada um dos recursos, e por consequência de todo o sistema, é impactada diretamente por um conjunto de variáveis, dos mais variados tipos, e que fazem com que o fluxo de caminhões ou a capacidade de carga e descarga varie, acarretando em mais ou menos filas dentro da planta. E, dependendo da combinação entre cada uma delas, o efeito causado no TPV (tempo de permanência de veículo) é significativamente alto.

Apesar de existir várias suposições por parte da equipe do projeto e das pessoas envolvidas nos processo sobre quais seriam estas variáveis, foi levantada uma base histórica para que se pudesse confirmar tais palpites e definir a lista de variáveis que seriam estudas e serviram de input para a simulação.

Finalizado os levantamentos, chegou-se a uma lista sobre quais seriam os fatores que poderiam impactar na eficiência desse processo. São elas:

- Concentração da chegada de caminhões: varia em função do dia do mês, do dia da semana e da hora do dia, conforme demonstrado na Figura 7;

- Composição de carga: necessidade de carregamento em mais de uma doca (famílias de produto diferentes dentro de um mesmo transporte);

- Tipo de caminhão: para o caso de inbound, se o veículo utilizado era tipo carga seca ou basculante, e no caso de produto acabado, se era utilizado carreta simples, carreta vanderléia, bitrem, rodotrem, etc;

- Disponibilidade de recursos: disponibilidade de recursos (equipamentos e pessoas) e qual o equipamento utilizado, sendo no caso de inbound, utilização de pontes rolantes e/ou escavadeiras tipo garras ou eletroímã, e no caso de outbound, utilização de ponte rolante com eletroímã, ponte rolante com ganchos ou empilhadeiras. 
Figura 7 - Curva percentual da chegada de veículos por dia da semana

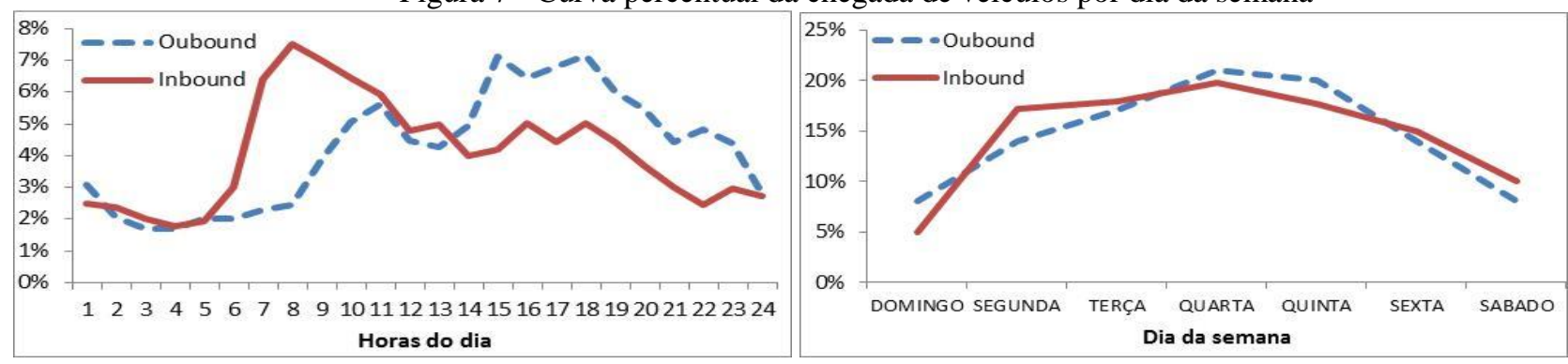

Fonte: Pesquisa de campo (2014)

\subsection{Cenários de análise}

Para este estudo, foram desenhadas duas configurações do sistema, sendo a primeira refletindo a configuração atual, onde existe uma única portaria e os fluxos de inbound e outbound compartilham os mesmos recursos (cadastro, preparação e balança, tanto na entrada como na saída), conforme Figura 3. A segunda configuração representa a simulação do processo com fluxos independentes através da construção de uma nova portaria, o qual está representado esquematicamente na Figura 8.

Figura 8 - Fluxo logístico proposto para os processos Inbound e Outbound

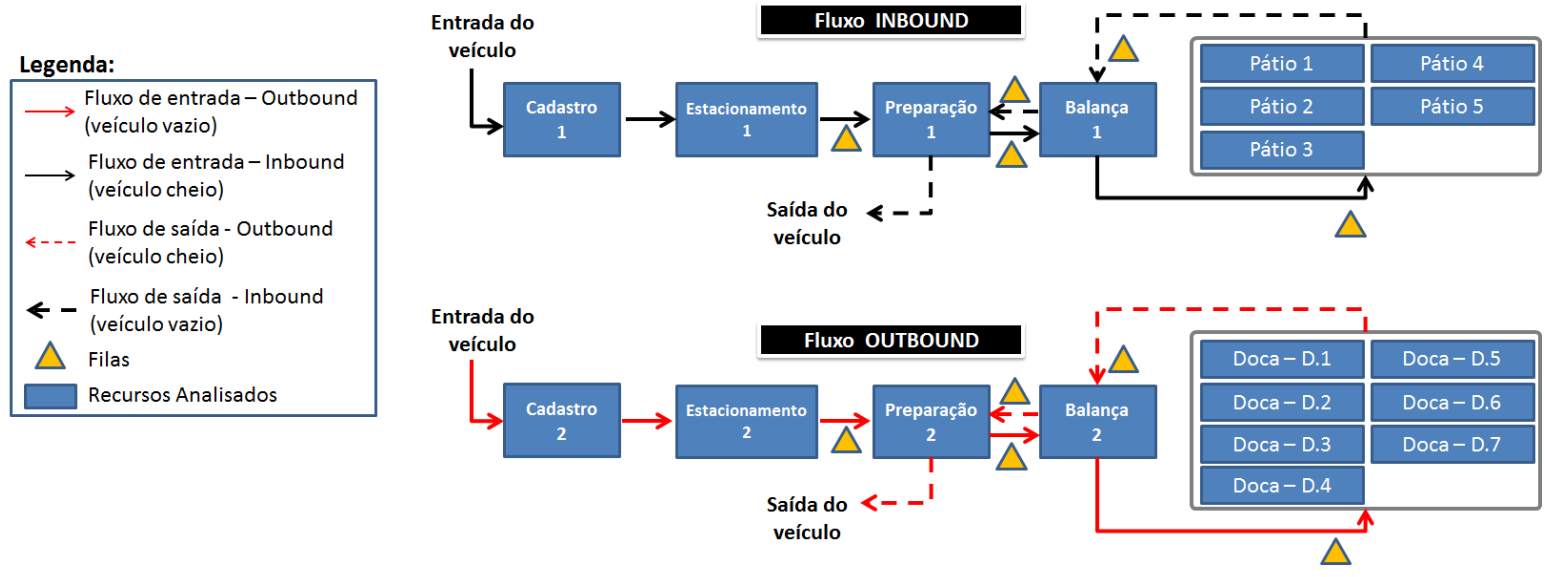

Fonte: Autoria própria (2014)

Em cada uma dessas configurações (modelos), foram desenhados cenários para simular a demanda atual e também a demanda projetada para os próximos anos (aumento de 10\%, $20 \%$ e $30 \%)$.

Para introduzir essa demanda no modelo de simulação, foi calculado o número de movimentação de veículos que entrariam e sairiam da planta em cada uma das configurações / cenários, conforme demonstrado na Figura 9, e que seriam utilizados como input para a simulação. 


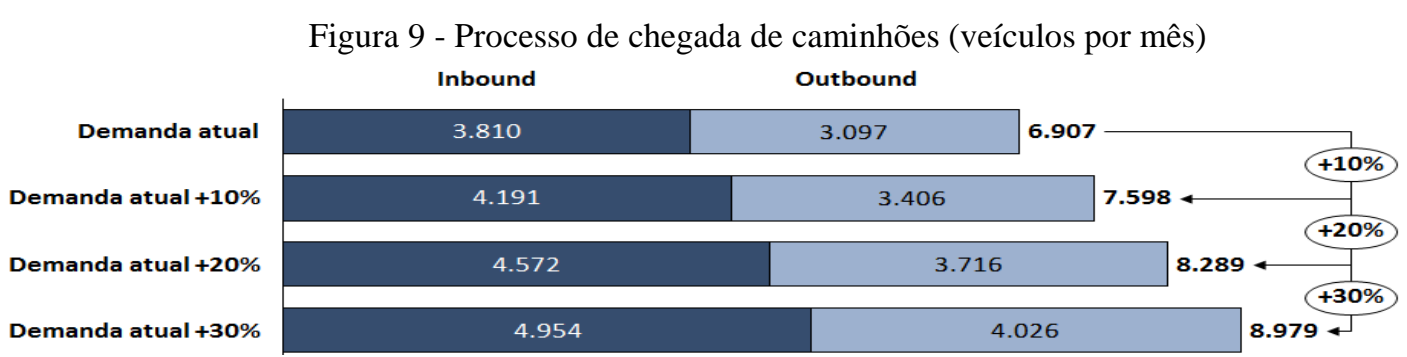

Fonte: Autoria própria (2014)

No modelo de duas portarias, em alguns cenários de aumento de demanda, foram criadas novas simulações com a adição de recursos extras em locais críticos do processo para que se conseguisse reduzir ainda mais o TPV, ou seja, além da separação dos fluxos estudou-se também o efeito da adição de capacidade nos gargalos do processo (aplicação da ToC).

\subsection{Modelagem e simulação dos cenários}

Diante do problema exposto, foram construídos modelos de simulação para representar as duas configurações (com uma e duas portarias), os quais foram representados no software ProModel. Para tal, foram utilizados todos os dados e variáveis previamente coletados, como a curva de chegada de veículos, o tipo de caminhão, a composição de carga de cada pedido, os tempos e movimentos de cada um dos recursos, tempos de parada, etc, os quais foram usados como dados de entrada do modelo.

Para a construção e validação da capacidade do modelo em refletir com a melhor acuracidade possível a realidade, foram seguidos os passos propostos por Harrel et al. (2004), conforme já descrito na fundamentação teórica.

Iniciou-se a construção pela criação das entidades (veículos de cada fluxo) e variáveis do modelo (mencionadas na seção 4.2), as quais ajudaram a refletir as aleatoriedades do sistema. Em seguida, foram criados os locais e os recursos necessários para cada etapa, como por exemplo, o cadastro, a balança, as docas, e todos os recursos da operação (headcount e equipamentos). $\mathrm{Na}$ sequência, foi feita toda a roteirização (processos) que cada veículo (entidade) teria que percorrer dentro da planta, e por fim, foram adicionadas as curvas de chegada para tipo de fluxo, respeitando as variações diárias e mensais.

Como os recursos utilizados na operação de carga e descarga são compartilhados com a produção, foram necessárias algumas simplificações para reduzir a complexidade da modelagem. Assim, baseado na tomada de tempos e movimentos, cada interferência no processo de carregamento era registrada (conforme Figura 5).

Esse registros foram submetidos a uma análise estatística através do programa StatFit (software auxiliar ao ProModel), o qual retorna a melhor curva que descreve a distribuição dos 
tempos deste processo. Tais curvas foram usadas para representar o tempo de carregamento em cada uma das docas, refletindo assim o processo como um todo, com suas médias e variações por causa das interferências. Estes mesmos registros, além de servirem como input para a simulação, também serão úteis para trabalhos futuros para redução de interferências.

Para ter um maior controle das filas do sistema, foram criadas variáveis para contar o número de veículos na fila em todas as etapas de cada um dos processos, de modo a facilitar a identificação dos gargalos para cada local / recurso.

O primeiro modelo foi desenhado respeitando o fluxo esquematizado na Figura 3. Para se ter uma melhor visualização da movimentação de caminhão dentro da planta, toda a modelagem foi construída utilizando-se a vista aérea da unidade, e, como exemplo do software de simulação, é apresentada na Figura 10 uma tela com a roteirização de todo o processo e suas lógicas.

Figura 10 - Tela exemplo com a roteirização do cenário com uma portaria

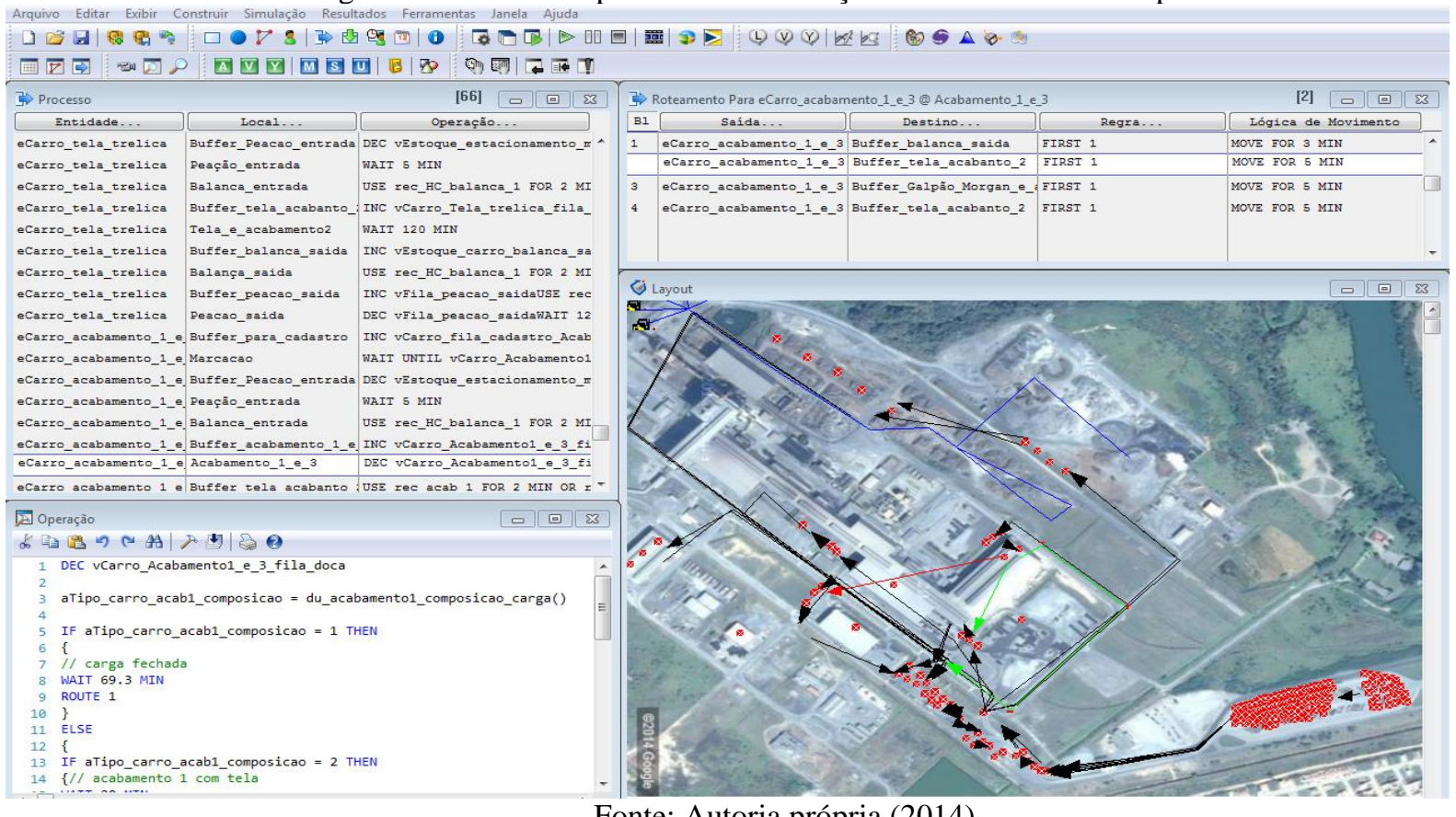

Fonte: Autoria própria (2014)

Como parâmetro de simulação foram feitas cinco replicações com um período de aquecimento de 720 horas (30 dias) para que a planta pudesse entrar em "regime permanente" e depois foram simuladas 4.320 horas (180 dias) para cada cenário de modo a se obter resultados confiáveis.

\subsection{Validação do modelo}

Para validar se o modelo desenvolvido representava com fidelidade a operação atual, foi comparado o TPV obtido através da simulação com o TPV real demonstrado na Figura 1. Tal comparação é mostrada na Figura 11. 


Figura 11 - Comparativo do TPV meta real x TPV obtido
\begin{tabular}{|c|c|c|c|c|}
\hline Processo & Mês & Real & Modelo & Desvio \\
\hline Inbound & mês 1 & $8: 37$ & $8: 47$ & $2 \%$ \\
\hline Inbound & mês 2 & $9: 26$ & $8: 41$ & $-8 \%$ \\
\hline Inbound & mês 3 & $10: 09$ & $9: 39$ & $-5 \%$ \\
\hline Inbound & mês 4 & $9: 05$ & $9: 22$ & $3 \%$ \\
\hline Inbound & mês 5 & $7: 56$ & $7: 32$ & $-5 \%$ \\
\hline Inbound & mês 6 & $8: 54$ & $8: 22$ & $-6 \%$ \\
\hline Inbound & mês 7 & $8: 12$ & $8: 02$ & $-2 \%$ \\
\hline Outbound & mês 1 & $4: 28$ & $4: 34$ & $2 \%$ \\
\hline Outbound & mês 2 & $5: 16$ & $4: 51$ & $-8 \%$ \\
\hline Outbound & mês 3 & $5: 42$ & $5: 49$ & $2 \%$ \\
\hline Outbound & mês 4 & $6: 05$ & $5: 50$ & $-4 \%$ \\
\hline Outbound & mês 5 & $5: 12$ & $4: 53$ & $-6 \%$ \\
\hline Outbound & mês 6 & $5: 34$ & $5: 44$ & $3 \%$ \\
\hline Outbound & mês 7 & $4: 50$ & $4: 38$ & $-4 \%$ \\
\hline
\end{tabular}

Fonte: Autoria própria (2014)

Os resultados apresentados na Figura 11 mostram que, apesar de pequenas variações existentes entre os dados reais e os resultados obtidos através da simulação, o modelo pôde ser validado, confirmando a aderência à realidade, servindo como base para a simulação dos demais cenários propostos. Como comentário, vale ressaltar que a maioria dos desvios entre o modelo e a realidade ocorre para menor devido a grande dificuldade de se reproduzir eventos anômalos e de difícil modelagem que paralisam a operação da planta por algumas horas, como falta de energia muito longa, chuvas torrenciais, queda do sistema por falta de rede, entre outros.

\subsection{Análise de cenários}

Conforme descrito anteriormente, iniciou-se a análise com as configurações pré-definidas de uma e duas portarias, variando-se a demanda. Entretanto, durante a simulação foi possível notar que para determinados cenários de demanda, a planta não suportava o volume de veículos, mesmo no modelo com duas portarias (portarias independentes). Dado este fato, simulou-se também o cenário de duas portarias com a adição de outros recursos extras identificados como gargalos durante a simulação.

No decorrer das simulações, para identificar se a planta possuía capacidade suficiente para atender a demanda a qual foi submetida, foi analisada a fila total do sistema. Na Figura 12, é apresentado o comparativo da demanda atual (esquerda) em relação ao cenário com 30\% de aumento. Pode-se observar que a fila da situação atual, apesar de alguns picos, encontra-se sob controle, e no cenário com $30 \%$ de aumento da demanda, a fila tem uma tendência de aumento ao longo do tempo, ficando evidente a falta de capacidade da planta em atender a esta demanda. 
Figura 12 - Número de veículos na fila do sistema: demanda atual (esquerda) x demanda com aumento de $30 \%$ (direita)
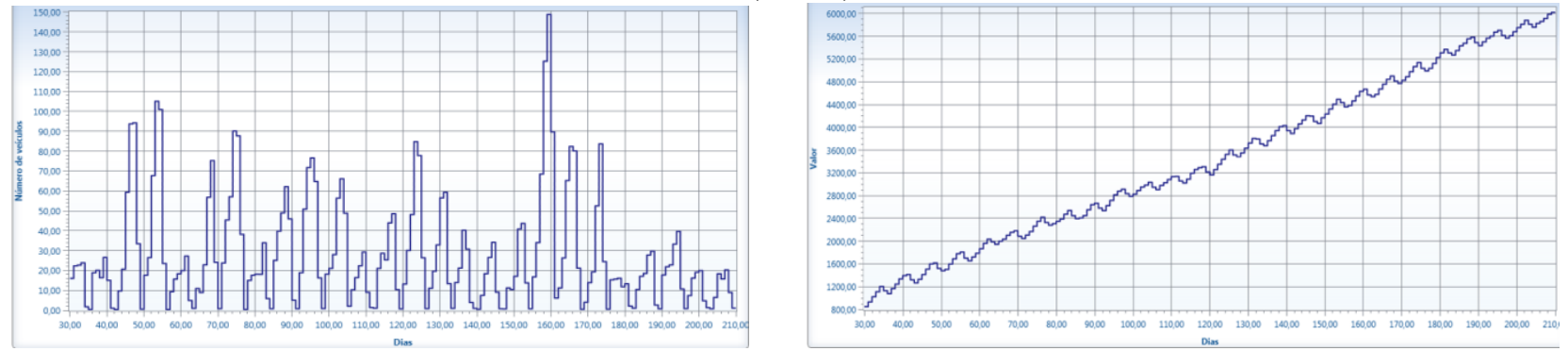

Fonte: Autoria própria (2014)

Uma vez observada a incapacidade de o sistema absorver o aumento de demanda, e seguindo as regras da teoria das restrições, foi preciso descobrir qual seria o local ou recurso mais crítico de cada processo (inbound e outbound) para que se pudesse simular um novo cenário com o aumento da capacidade destes gargalos. Para isto foram analisados a fila e o tack time de cada um desses locais/recursos. Na Figura 13, é exibida a análise das filas do processo outbound, onde ficou evidente que o novo gargalo estava na "Preparação de saída".

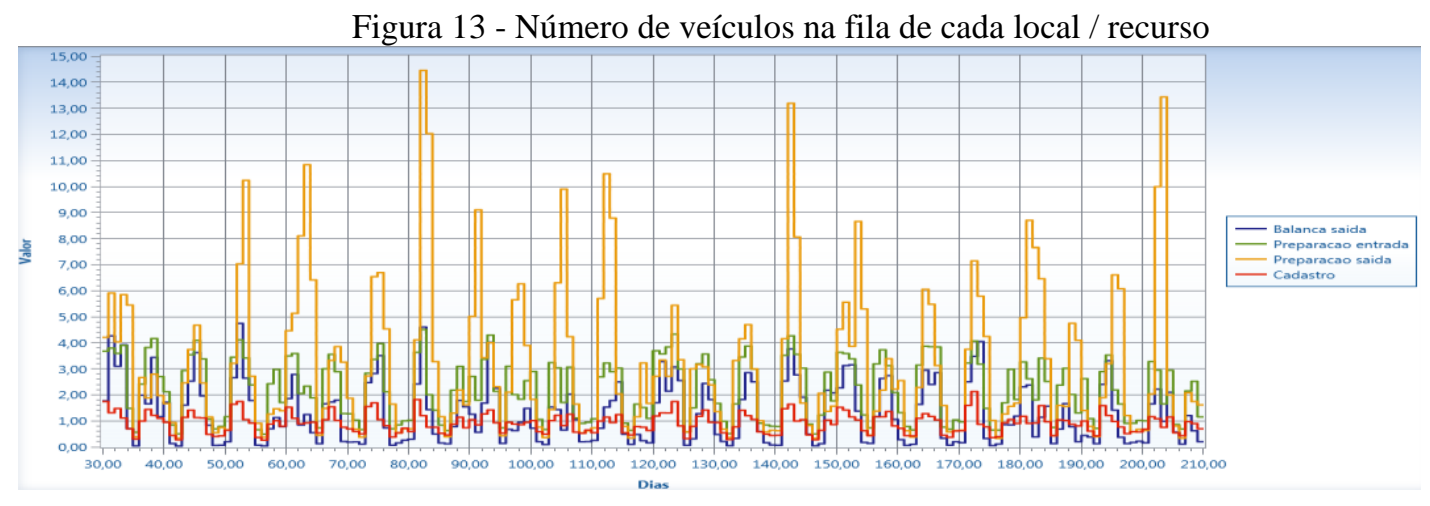

Fonte: Autoria própria (2014)

\section{Discussão dos Resultados}

O resultado final de todas as simulações e os comparativos de redução no TPV são apresentados na Figura 14. Nesta mesma figura, também são apresentadas mais duas informações, a primeira delas é referente aos gastos com estadias, que ocorrem em função do pagamento de diárias aos transportadores devido ao elevado número de horas que o veículo permanece dentro da usina. Este valor foi comparado ao cenário atual, o qual foi considerado como sendo a base 100, e os demais resultados uma comparação relativa de quais seriam os gastos estimados com estadias. A outra informação refere-se aos investimentos necessários para cada um dos cenários propostos, separados em Capex e Opex. 
Figura 14 - Resultados dos modelos de simulação

\begin{tabular}{|c|c|c|c|c|c|}
\hline Demanda & Cenário & Modelo & TPV total (hr) & $\begin{array}{c}\text { Tempo de } \\
\text { operação (hr) }\end{array}$ & $\begin{array}{c}\text { Tempo de Fila } \\
\text { (hr) }\end{array}$ \\
\hline \multirow{3}{*}{ Atual } & 1A & Uma portaria & 7,79 & 1,21 & 6,58 \\
\hline & $2 \mathrm{~A}$ & Duas portarias & 5,55 & 1,21 & 4,34 \\
\hline & & Redução 1A x 2A (\%) & $-29 \%$ & $0 \%$ & $-34 \%$ \\
\hline \multirow{3}{*}{$+10 \%$} & 1B & Uma portaria & 12,72 & 1,21 & 111,51 \\
\hline & $2 \mathrm{~B}$ & Duas portarias & 6,79 & 1,21 & 5,58 \\
\hline & & Redução 1B x 2B (\%) & $-47 \%$ & $0 \%$ & $-52 \%$ \\
\hline \multirow{4}{*}{$+20 \%$} & $1 \mathrm{CC}$ & Uma portaria & \multicolumn{3}{|c|}{ Cenário não factivel - falta de capacidade dos recursos } \\
\hline & $2 \mathrm{C}$ & Duas portarias & 14,93 & 1,21 & 13,72 \\
\hline & 2C_v2 & Duas portarias + recurso extra & 4,49 & 1,21 & 3,28 \\
\hline & & Redução 2C x 2C_v2 (\%) & $-70 \%$ & $0 \%$ & $-76 \%$ \\
\hline \multirow{4}{*}{$+30 \%$} & $\begin{array}{l}\text { 1D } \\
\text { 2D }\end{array}$ & $\begin{array}{c}\text { Uma portaria } \\
\text { Duas portarias }\end{array}$ & \multicolumn{3}{|c|}{$\begin{array}{l}\text { Cenário não factível - falta de capacidade dos recursos } \\
\text { Cenário não factível - falta de capacidade dos recursos }\end{array}$} \\
\hline & 2D_v2 & Duas portarias + recurso extra & 8,33 & 1,21 & 7,12 \\
\hline & 2D_v3 & Duas portarias +2 recursos extras & 3,67 & 1,21 & 2,46 \\
\hline & & Redução 2D_v2 X 2D_v3 (\%) & $-56 \%$ & $0 \%$ & $-66 \%$ \\
\hline
\end{tabular}
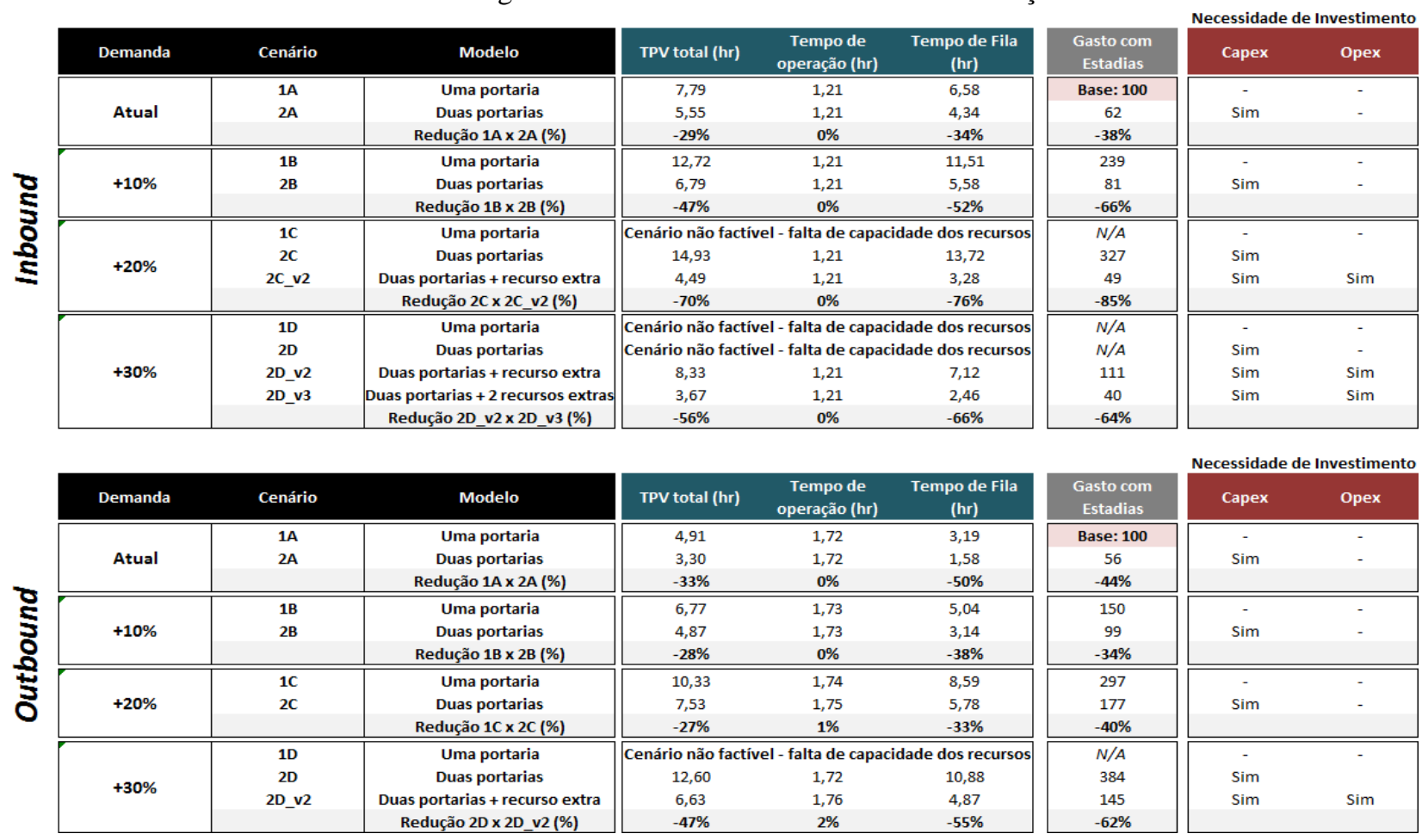

Fonte: Autoria própria (2014)

Analisando os resultados apresentados na Figura 14, é possível notar o impacto significativo que existe no TPV e nas filas mesmo quando se adiciona apenas uma pequena variação de demanda. Isso se deve ao fato de a planta já estar trabalhando próxima de seu limite operacional, com uma taxa de utilização de seus recursos muito elevada.

Em qualquer um dos cenários de demanda testados, seja para inbound ou outbound, o modelo com portarias independentes (duas portarias) traz melhoras significativas para a redução o tempo de permanência de veículos na planta, variando de uma redução de $27 \%$ a até $47 \%$, o que mostra o efeito bastante positivo em se separar estes dois fluxos.

Com o gradual aumento da demanda, alguns cenários se tornaram inviáveis de serem realizados, dada a falta de capacidade dos recursos em suportar a demanda ao qual foram submetidos, como mostrado nos cenários de 1D para outbound e 1C, 1D e 2D para inbound.

Em alguns cenários, como por exemplo, o cenário 2D para outbound, o sistema possui capacidade suficiente para suprir toda a demanda, porém, o TPV se torna tão elevado que os gastos com estadias triplicariam em relação ao atual (vide coluna "Gasto com estadias" da Figura 14). Assim seja pela falta de capacidade do sistema ou pelo alto TPV associado a determinado cenário, decidiu-se simular cenários alternativos com a adição de capacidade nos recursos críticos do sistema (gargalos), conforme já explicado anteriormente.

A adição de recursos extras traz resultados igualmente significativos quando comparados aos resultados obtidos da adoção de portarias independentes, e a exemplo do cenário com $+30 \%$ de 
demanda de inbound, a combinação entre o modelo de duas portarias e o da adição de novos recursos se tornam imprescindíveis para a viabilidade da operação.

No entanto, apesar das grandes reduções no TPV proporcionadas pela separação dos fluxos e/ou pela adição de recursos extras, toda essa melhoria exige investimentos, sejam eles Capex ou Opex, conforme identificado na coluna "Necessidade de investimento" da Figura 14.

Dessa forma, apesar dos investimentos necessários não estarem expressos em montante, a recomendação deste trabalho é que seja feito de imediato a separação dos fluxos inbound e outbound, de modo que se tenha duas portarias independentes, o que apesar do valor a ser investido, trará resultados significativos na redução dos gastos com estadias pagos as transportadoras.

Num segundo momento, quando a demanda atingir $20 \%$ acima da atual, recomenda-se a adição de um recurso adicional para a descarga do fluxo inbound, e, posteriormente quando se atingir uma demanda $30 \%$ acima da atual, recomenda-se a adição de mais um recurso para inbound e a também de mais um recurso no fluxo outbound, mais especificamente na preparação de saída, conforme já mencionado na seção anterior e ilustrado da Figura 13.

\section{Conclusões}

Este trabalho foi desenvolvido com o objetivo de determinar a melhor configuração para os fluxos inbound e outbound para suportar o crescimento projetado de vendas/operações nos próximos 5 anos. Como conclusão, foi elaborada uma lista de recomendações de ações e investimentos que deveriam ser feitas para que a planta em análise possua totais condições de atender a demanda futura, do melhor modo, e na menor configuração de custo.

Iniciou-se o trabalho com a tomada dos tempos em cada uma das operações, passando pela análise histórica de todas as variáveis que poderiam exercer alguma influência sobre o sistema, e finalizando com a simulação de diversos cenários, de layout e demanda, a fim de avaliar qual seria o comportamento da planta perante aquelas variáveis de entrada.

A simulação das operações de carregamento e descarregamento através do software ProModel se mostrou bastante eficiente em retratar com elevado grau de acuracidade a realidade da planta, possibilitando a empresa apostar e investir nos resultados obtidos e na lista de recomendações sugeridas.

Como sugestão, é recomendada uma análise dos fatores que causam as concentrações de chegada dos veículos, seja ela durante um único dia ou mesmo ao longo do mês, que envolve fatores de mercado, podendo neste campo, se propor uma programação de chegadas que seja mais inteligente e nivelada, como por exemplo, o agendamento de horário, modalidade esta já realizada por diversas empresas. 
Por fim, como próximos passos, recomenda-se que seja feito o modelo econômico deste projeto, analisando e detalhando os investimentos necessários, comparando-os com os ganhos apresentados. Além disso, recomenda-se também que este estudo seja replicado para as demais plantas da empresa para que a mesma, além de garantir o pleno atendimento a demanda do mercado, possa fazê-lo com o menor investimento possível, atuando nos gargalos das operações logísticas dos fluxos inbound e outbound e reduzindo o tempo de permanência de veículos dentro da unidade.

Este trabalho limitou-se em estudar o fluxo logístico da unidade, não tendo como parte do escopo simular as atividades de relacionadas a produção para identificar gargalos no processo produtivo quando submetidos a demandas superiores a atual. Esta etapa será desenvolvida em um trabalho posterior a este, e utilizará a mesma metodologia aqui apresentada.

\begin{abstract}
The objective of this document is to present the case study of the layout change in logistics operations of an industrial company and the scenarios simulation, with its impacts and proposals of solutions. Due to resources sharing in the inbound and outbound process in one of the company's plant, the time that each vehicle remains within the unit (called TPV) was too high, impacting customer services and generating a massive spending with the payment to the carriers due to this high time, scenario that could get worst because the expected production growth for the next years. Thus, the purpose of this study is to define by simulation what is the best configuration of inbound and outbound flows to support the projected growth of sales / operations over the next five years and reduce the time that the vehicles remains inside the plant. Through the simulation process, it was possible to develop some scenarios with layouts changes and another ones adding new features, which generated reduction up $70 \%$ in the TPV and consequently had the reduction in spending money. Therefore, this work resulted in a list of recommendations to improve the logistics operation efficiency and customer service.
\end{abstract}

Key-words: capacity planning; modeling; logistics simulation; supply chain.

\title{
Referências
}

BALLOU, R. Gerenciamento da cadeia de suprimentos/logística empresarial. Porto Alegre: Bookman, 2006.

BATEMAN, R.; BOWDEN, R.; GOGG, T.; HARREL, C.; MOTT, J.; MONTEVECHI, J. A. B. Simulação de sistemas: aprimorando processos de logística, serviços e manufatura. 1ed. Rio de Janeiro: Elsevier, 2013.

CORRÊA, H. L.; GIANESI. I. G. N.; CAON, M. Planejamento, Programação e Controle da Produção, 5ed. São Paulo: Atlas, 2010.

COX III, J. F.; SPENSER, M. S. Manual da teoria das restrições. Porto Alegre: Bookman, 2002.

IANNONI, A.P.; MORABITO, R. Análise do sistema logístico de recepção de cana de açúcar: um estudo de caso utilizando simulação discreta. Gestão \& Produção, v.9, n.2, p. 107-128, São Carlos, 2002.

GOLDRATT, E. M.; COX Jeff. A Meta - um processo de melhoria contínua. 2ed. São Paulo: Nobel, 2003.

HARREL, C. R.; GHOSH, B. K.; BOWDEN, R. Simulation using Promodel. 2ed. New York: McGraw-Hill, 2004. 
KRAJEWSKI, L., RITZMAN, L., MALHOTRA, M. Administração da Produção e Operações, 8ed. São Paulo: Pearson Prentice Hall, 2009.

LONGO, F. Advances of modeling and simulation in supply chain and industry. Simulation, v. 87, n. 8, p. 651-656, 2011. cross ref

MOEllmanN, A. H. Aplicação da Teoria das Restrições no Gerenciamento da Cadeia de Suprimentos. 2008. 163f. Dissertação (Mestrado) - Faculdade de Engenharia do Campus de Guaratinguetá, Universidade Estadual Paulista, Guaratinguetá, 2008.

OLIVEIRA, C.M. Desenvolvimento de um sistema de simulação para Cadeias de Suprimentos. 2004. Tese (Doutorado) - Faculdade de Engenharia Mecânica, Universidade Estadual de Campinas, Campinas, 2005.

SAHINOGLU, M. Modeling and simulation in engineering. Wiley Interdisciplinary Reviews: Computational Statistics, v. 5, n.3, p.239-266, 2013. crossref

SELliTTO, M. F.; BORCHARDT, M.; PEREIRA, G. M. Análise de uma operação logística de carregamento e expedição de cimento por simulação computacional. Revista Gestão Industrial, v. 5, p. 130-151, 2009. cross ref

SLACK, N.; STUART, C.; JOHNSTON, R. Administração da Produção. São Paulo: Atlas, 2009.

\section{Dados do autor}

Nome completo: Daniel Bovo

Filiação Institucional: Universidade de São Paulo - USP

Função ou cargo ocupado: Consultor de Supply Chain

Endereço completo: Rua Ciro Lagazzi, № 1, Jardim Cândida, Araras-SP. CEP: 13603-027.

Telefone para contato: (11) 963502148

e-mail: danielbovo@hotmail.com

Submetido em: 16-03-2015

Aceito em: 14-09-2015 